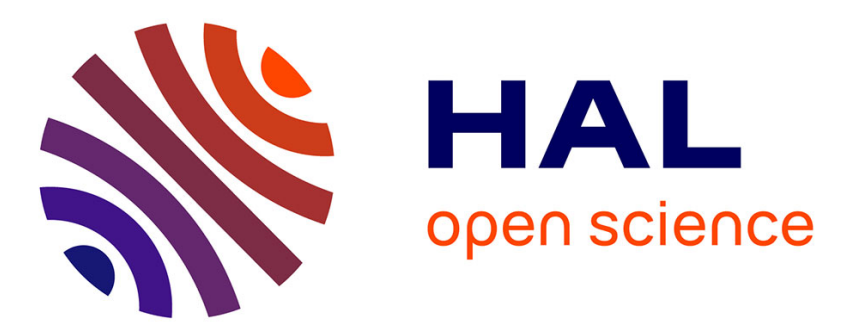

\title{
Real-time algorithm for versatile displacement sensors based on self-mixing interferometry
}

\author{
Antonio Luna Arriaga, Francis Bony, Thierry Bosch
}

\section{To cite this version:}

Antonio Luna Arriaga, Francis Bony, Thierry Bosch. Real-time algorithm for versatile displacement sensors based on self-mixing interferometry. IEEE Sensors Journal, 2016, 16 (1), pp.195-202. 10.1109/JSEN.2015.2478755 . hal-01226909

\section{HAL Id: hal-01226909 \\ https://hal.science/hal-01226909}

Submitted on 10 Nov 2015

HAL is a multi-disciplinary open access archive for the deposit and dissemination of scientific research documents, whether they are published or not. The documents may come from teaching and research institutions in France or abroad, or from public or private research centers.
L'archive ouverte pluridisciplinaire HAL, est destinée au dépôt et à la diffusion de documents scientifiques de niveau recherche, publiés ou non, émanant des établissements d'enseignement et de recherche français ou étrangers, des laboratoires publics ou privés. 


\title{
Real-time algorithm for versatile displacement sensors based on self-mixing interferometry
}

\author{
Antonio Luna Arriaga, Francis Bony, and Thierry Bosch, Senior Member, IEEE
}

\begin{abstract}
This paper presents a general displacement reconstruction algorithm for sensors based on self-mixing (SM) interferometry suitable to work at different optical feedback conditions. The approach relies on a robust phase observation through analytic signals for the fringe detection stage, while the motion of the pointed target is retrieved by a self-adapting filter in a piece-wise basis. The implementation for real-time calculations demonstrates the feasibility of robust SM sensors for different usage conditions without the need of modifying the base device configuration.
\end{abstract}

Index Terms-Self-mixing; displacement measurement; instrumentation and measurement.

\section{INTRODUCTION}

$\mathbf{T}$ HE attributes of compactness, low-cost and self-aligned nature of self-mixing interferometry, make this sensing scheme an attractive solution to assist several research domains as can be appreciated in extensive revisions of its applications (e.g. absolute distance, displacement and velocity) [1].

The physical principle of SM interferometry consists in the generation of interferences between an emitted beam and a small amount of backscattered light from a remote target, inside the active cavity of a laser. When a laser diode (LD) is used, the generated optical output power variations (OOP) can be monitored through its built-in photodiode or directly from the junction voltage. This base configuration reveals the fully integrated nature of SM sensors since the LD acts as source and micro-interferometer at the same time. In particular, when the laser beam is aimed to a moving target, the retrieved fringe pattern is related to the amount of displacement and its direction on the optical axis.

SM theory defines a feedback coupling factor $C$ notably related to the effective power reflectivity of the illuminated target as well as the distance of the external cavity created between its surface and the LD. Three main optical feedback regimes can be identified through this value: weak $(0.1<$ $C<1)$, moderate $(1<C<4.6)$ and strong $(C>4.6)$. Sinusoidal or asymmetric SM interferometry fringes devoid of sharp discontinuities are characteristic of weak feedback regime. Sawtooth-like SM interferometry fringes exhibiting hysteresis are proper of moderate feedback, while in the strong optical feedback, higher values of $C$ lead to a chaotic SM signal [2].

To treat this complex time-varying shape of the fringes (whose spectral properties are also affected), it is common

The authors are with the CNRS, LAAS, 7 avenue du colonel Roche, F31400 Toulouse, France and the Univ de Toulouse, INP, LAAS, F-31400 Toulouse, France (e-mail: alunaarr@laas.fr). to observe in literature a restriction on the feedback regime for specific applications. As a matter of fact, even minor usage modifications usually require a complete redesign of the instrument [1]. By using external components to the base SM configuration it is possible to perform simple signal processing. More elaborated algorithms alleviate this constraint, however a tradeoff between complexity and robustness of signal exploitation needs to be addressed. For the first case, in [3] an electro-optical modulator was used to implement a quadrature demodulation allowing displacement reconstruction of SM signals in $C<1$. Rather in [4] it was proposed to retrieve displacement by locking a moderate SM regime with a specific optical setup and an electronic feedback loop control. Recently, a real-time calculation method of $C$ combined with an autofocus via an adaptive liquid lens demonstrated the feasibility of autonomous SM displacement sensors by preventing the signal to quit a moderate optical feedback regime [5]. In the second case, the method from [6] required a time consuming estimation of $C$ and calibration prior to perform a displacement reconstruction. [7] demonstrated real-time calculations by selecting a convenient algorithm to process a detected feedback regime at poweron. However this system requires a manual reset when optical conditions change and its resolution changes by almost half of the LD's wavelength between the detected regimes. Two other correlated algorithms, the phase unwrapping method (PUM) [8] and the adaptive threshold algorithm (ATA) [9] are discussed in next section to highlight the contribution of our proposal.

Recently we reported a robust phase calculation to detect SM fringes randomly affected in amplitude due to speckle phenomenon [10]. Since performing displacement reconstruction mainly relies on properly detecting SM fringes, in this work our interest is to provide a unique algorithm to perform displacement reconstruction from SM signals of different feedback regimes disregarding the coupling factor $C$. The proposed approach avoids the need to lock a preferable working mode by external components and avoids the need of switching between different calculation methods. By doing so, the algorithm shall be implemented into different processing architectures allowing to meet timing constraints for versatile SM sensors.

\section{RELATED WORK ON SM SIGNAL EXPLOITATION}

Two equations largely referenced in SM literature (e.g. [2]) describe the main relationship between the monitored OOP variations $P(t)$ and its phase:

$$
P(t)=P_{0}\left\{1+m \cos \left[x_{f}(t)\right]\right\}
$$




$$
x_{0}(t)=x_{f}(t)+C \sin \left[x_{f}(t)+\arctan (\alpha)\right]
$$

where $P_{0}$ is the optical power under free-running conditions, $m$ a modulation index, $x_{f}$ the phase of the signal subject to back-reflections, $x_{0}$ the original phase and $\alpha$ the LD's linewidth enhancement factor. Written as a function of the wavelength subject to back-reflections $\lambda_{f}(t)$ and the instantaneous distance to source $D(t)$, the monitored signal phase $x_{f}(t)=4 \pi D(t) / \lambda_{f}(t)$ allows to observe that target's displacement can be obtained from (2) by calculating $\alpha$ and C.

\section{A. PUM and the adaptive threshold improvement}

Displacement reconstruction with PUM can be achieved by normalizing the signal from (1) $\left[P_{N}=P(t) / P_{0}\right]$ in order to estimate a rough phase via an $\arccos$ function:

$$
\hat{x}_{f}(t)=\arccos \left[P_{N}(t)\right]
$$

By using (3) on this normalized signal, a mirrored phasemapped calculation is obtained with a twofold purpose: 1) it allows to detect positive and negative discontinuity points by its derivative, and 2) it enables displacement reconstruction by an optimization criteria which calculates $C$ and $\alpha$ from the unwrapped phase.

To illustrate PUM method, Fig.1 presents a simulated signal obtained by the behavioral model from [2]. The target displacement corresponds to a sinusoidal period with amplitude of $10 \lambda_{0}$ peak-to-peak (p-p), being $\lambda_{0}$ the wavelength of a LD. In Fig.1(b) it can be appreciated that the motion of the pointed target can be retrieved from this phase-mapped signal if SM fringes are properly identified in a preliminary step.

In order to detect fringes for different feedback regimes, PUM was improved in [9] by introducing an adaptive threshold over the arccos derivative step. The iterative loop from ATA discriminates a particular feedback regime to perform specific filtering procedures for displacement reconstruction.

Considering that displacement reconstruction step in PUM involves time-consuming calculations for $C$ and $\alpha$ [11], it can be foreseen that the implementation of a complete

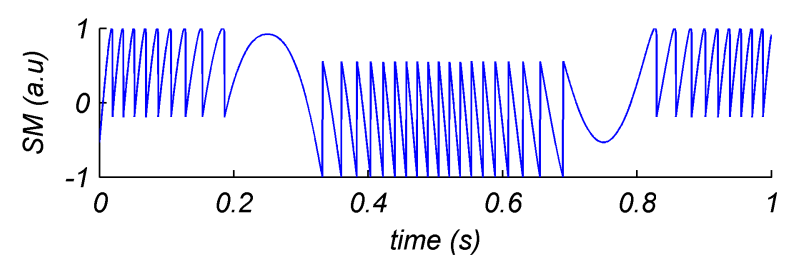

(a) SM with $C=3$ for a displacement amplitude of $10 \lambda_{0}$ p-p.

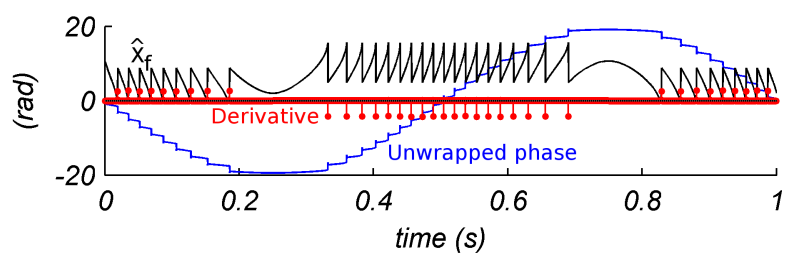

(b) Unwrapping detected fringes from $\hat{x}_{f}$ leads to target displacement.

Fig. 1. PUM applied to a simulated SM signal for one period of displacement.

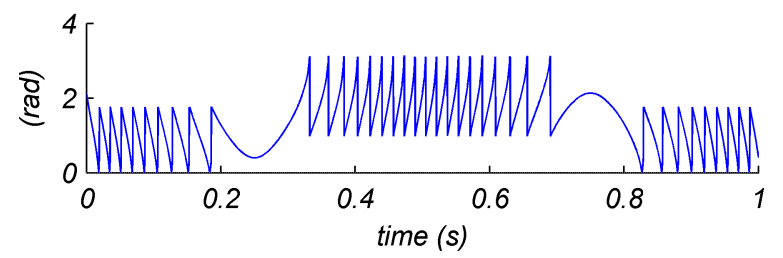

(a) Phase calculation by arccos from (3).

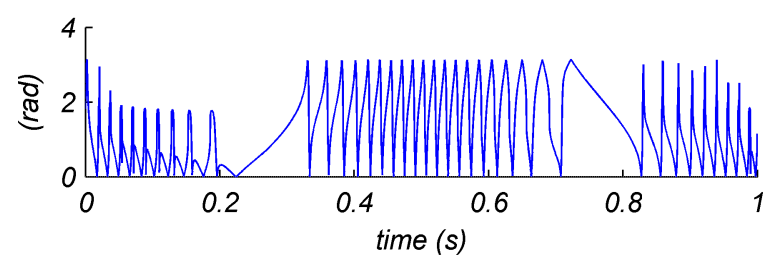

(b) Phase calculation by HT from (4).

Fig. 2. Comparison between conventional approach and the HT based proposal.

detection-reconstruction algorithm for real-time calculation shall be compromised by adding more elaborated steps to the derivative-based fringe detection step of PUM. Therefore, combining ATA for the detection stage with PUM for the reconstruction shall result in expensive computations that reduce the potential of implementing this solution as a sensor.

Let us now outline the theory of our fringe detection scheme based on the robust phase calculation via the Hilbert transform (HT) as detailed in [10].

\section{B. Hilbert transform based detection method}

One of the usefulness of analytic signal representation can be appreciated through the calculation of the instantaneous frequency as it provides a parameter which accounts the time-varying nature of a process. In the context of SM interferometry signals, HT allows to generate the orthogonal representation of the original OOP over the imaginary plane by providing a $90^{\circ}$ phase shift. This calculation can be seen as a filtering process between the kernel $(-\pi t)^{-1}$ and the signal of interest. The analytic form of an acquired SM signal, i.e. $P_{a}(t)=P(t)+\operatorname{Im}\{\mathrm{HT}[P(t)]\}$, allows to calculate the instantaneous phase by:

$$
\phi(t)=\arctan \left\{\frac{\operatorname{Im}\left[P_{a}(t)\right]}{\operatorname{Re}\left[P_{a}(t)\right]}\right\}
$$

In order to approach a linear representation of a fringe and its unwrapped phase of $2 \pi$, a four-quadrant arctan function can be used via the well known arctan 2 calculation which considers the sign of members in (4). As represented in Fig.2, the absolute value of a phase calculation by this proposal provides a similar observation than the one obtained by PUM for the simulated signal in Fig.1(a). Notice however that by using (4), the normalization step is not necessary, allowing to implement a system without automatic gain control.

\section{REAL-TIME DETECTION-RECONSTRUCTION ALGORITHM FOR WEAK AND MODERATE FEEDBACK}

This section details our proposed approach to perform the two major tasks on SM signal exploitation for displacement 


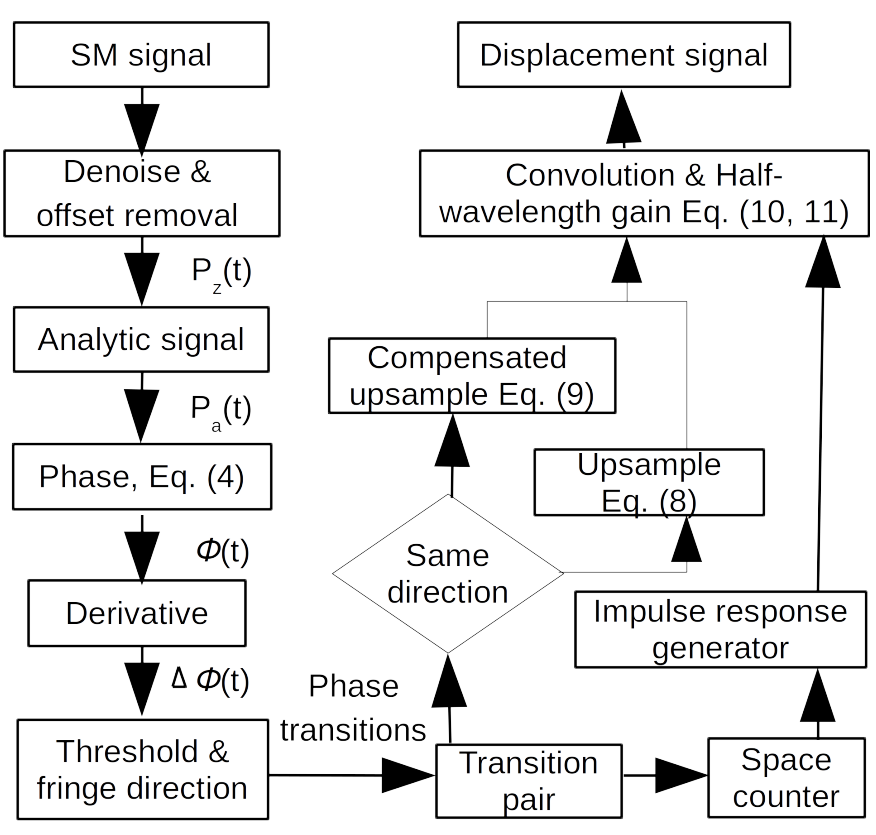

Fig. 3. Proposed real-time algorithm for versatile SM displacement reconstruction.

measurement: the fringe detection step and the displacement reconstruction with sub-wavelength resolution. The block diagram of Fig. 3 considers a digitized SM signal from a LD as input and provides the motion of a pointed vibrating surface as output. Just like in any signal acquisition system, signal noise needs to be removed at this level. In our current implementation, an averaging filter is used after acquiring a segment of SM signal.

\section{A. Fringe detection}

This is the cornerstone of SM interferometry. Since a target's displacement of $\lambda_{0} / 2$ in amplitude leads to the appearance of one fringe, an accurate measurement requires to detect all the acquired fringes. From the work presented in [10], let us elaborate here the strategy to obtain the calculations within time boundaries that satisfy the constraint for real-time processing. Three fundamental tasks represent this proposal: 1) offset removal, 2) phase calculation through analytic signal, and 3) transition's train generation.

1) Offset removal: $\mathrm{SM}$ signals need to be kept centered to the zero-axis for a proper orthogonal generation. We propose to locally remove hysteresis phenomenon from SM fringes by differentiation of the input signal. As depicted in Fig.4(a) and in a similar manner as PUM's detection step, a threshold can be used over the differentiated signal to detect most of the acquired fringes. In our case this is used as an approximation and not as a final calculation, therefore the detected fringes are referred here as pseudo-fringes. These values are used as trigger points to generate an average signal running along the fringes [Fig.4(b)]. This signal is then subtracted to the original SM segment in order to obtain a zero-centered set of SM fringes.

This procedure works also for the case of weak regime since the threshold is calculated by the root-mean-squared value

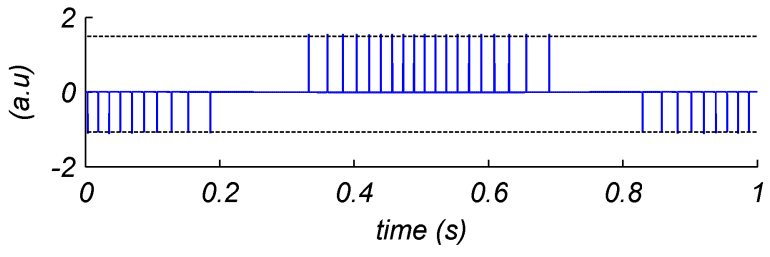

(a) Thresholding a differentiated SM signal segment.

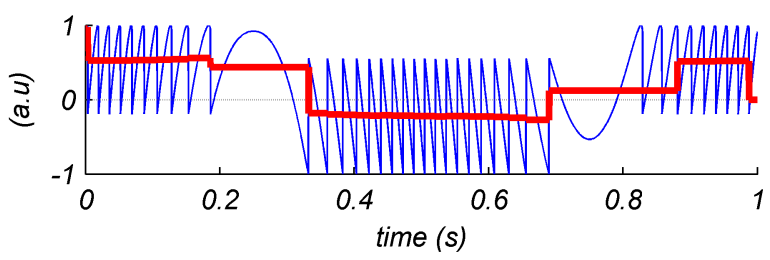

(b) Running average triggered by pseudo-fringes.

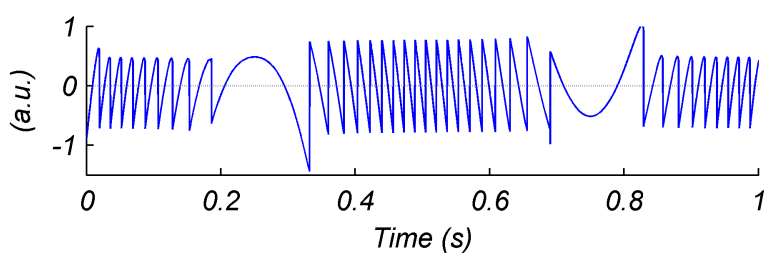

(c) Zero-centered set of SM fringes.

Fig. 4. Fringe offset removal step for the simulated signal in Fig.1(a) $(C=3)$.

of the positive and negative values of the signal. By using this approach the averaged signal to subtract is already close to zero along the time axis, resulting thus in a non-intrusive calculation for the moderate regime.

2) Phase calculation through analytic signal: The calculation of analytic SM signals can be directly obtained by using a Fast Fourier Transform (FFT) and its inverse (IFFT) via [12]:

$$
P_{a}(t)=I F F T \begin{cases}2 . F F T[P(t)], & t=(0, T / 2-1) \\ 0, & t=(T / 2, T-1)\end{cases}
$$

In Fig.5 it can be appreciated in an extended range, the homogeneous phase calculated from the analytic SM signal obtained from the zero-centered segment of Fig.4(c). Notice how this result contrasts the phase calculation by $\operatorname{abs}(\arctan 2)$ of the hysteresis-affected SM signal represented in Fig.2 which is equivalent to the previous referenced PUM phase estimation. The presence of small peaks (zoomed area) surrounding a peak of $2 \pi$ amplitude, denote also the moderate regime of the SM input signal $(C=3)$. By settling a fixed threshold to select these major peaks, a robust fringe detector is obtained as the phase pattern is similar between different feedback regimes, thus disregarding the shape in time of SM fringes.

3) Transition's train generation: From the obtained phase transitions corresponding to detected fringes, the remaining point consists in assigning their direction to retrieve a target's movement. One approach consists in reusing the differentiation step from the offset removal procedure to save on calculation resources. This signal is converted into positive and negative unitary pulses according to the opposite sign of each of its samples. 


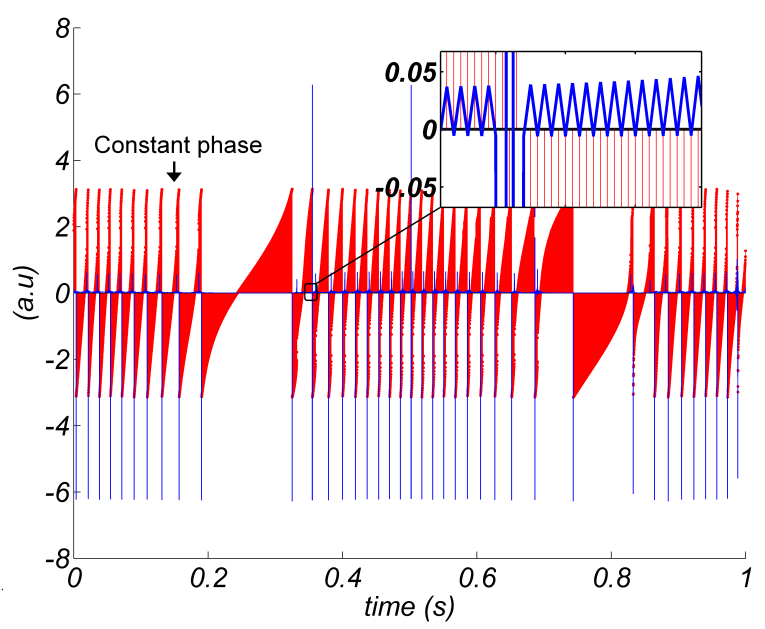

Fig. 5. Instantaneous frequency calculation for the simulated signal in Fig.1(a) $(C=3)$.

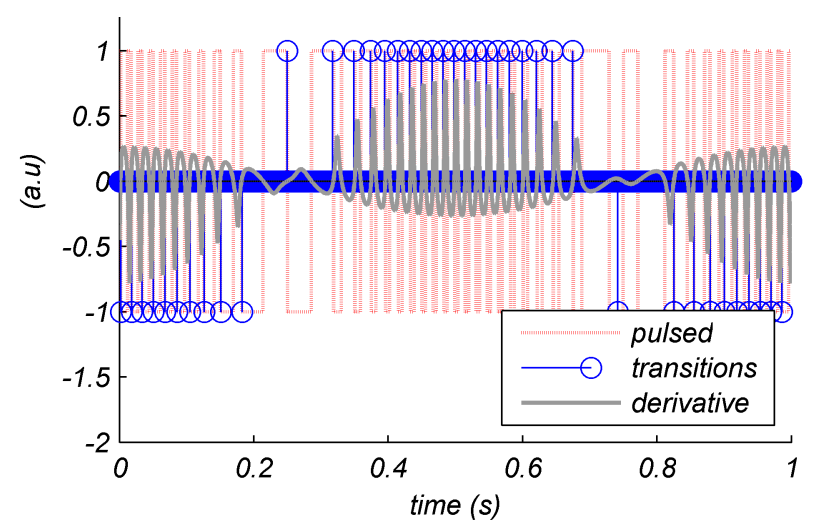

Fig. 6. Fringe direction assignation for a simulated signal with $C=0.5$ for a $10 \lambda_{0}$ p-p displacement amplitude.

As shown in Fig.6 the train of pulsed values for a signal in weak feedback $(C=0.5)$ provides the target's direction of each sharped derivative point, resulting in a valid calculation even in the case of moderate feedback [e.g. Fig.4(a)]. These pulsed values are then multiplied by the instantaneous frequency peaks denoted in Fig.5 to obtain the assigned direction for each phase transition.

\section{B. Displacement reconstruction}

Reconstruction step can begin once that SM fringes have been detected and assigned according to the target's direction. Since that first block demanded relatively complex calculations, we decided to simplify this second step at expenses of lower resolution.

Optimization criterion from PUM proposal used notably to estimate $C$, provides a reconstructed displacement with resolution of about $40 \mathrm{~nm}$ [8]. Conversely, unrolling a set of detected fringes provides a resolution of half-wavelength ( $\approx 390 \mathrm{~nm}$ for the LD used in our experiments) without the need of further calculations.

As depicted in Fig.7, reaching sub-wavelength resolution from SM signals at different feedback regimes is a com- plex task even considering that all the fringes have been detected properly. Since the fringes appear as a function of displacement and not as function of time, SM displacement reconstruction can be stated as a problem of waveform reconstruction from irregular samples. However, as the amount of fringes provides a good representation of a target's movement, it can be conceivable to achieve displacement reconstruction in a similar manner than a Digital-to-Analog (D/A) circuit naturally interpolates samples.

By using the equation of an ideal interpolator to model a D/A conversion process, equally spaced input samples $x(k)$ can be related with a time domain output signal $x_{\mathrm{a}}(t)$ by:

$$
x_{a}(t)=\sum_{k=-\infty}^{\infty} x(k) g_{a}(t-k T)
$$

where the convolution kernel $g_{a}(t)=\sin (\pi t / T) \pi t / T$ is a normalized cardinal sine (sinc) with rectangular transfer function, thus denoting an ideal low-pass filter [13].

Instead of using a sinc convolution kernel which provides a smooth reconstruction of the output signal, linear interpolation can be achieved by using a triangular impulse response (TRI):

$$
\operatorname{TRI}(t)= \begin{cases}t / T, & 0 \leq t<T \\ 2-t / T, & T \leq t<2 T \\ 0, & \text { otherwise }\end{cases}
$$

Increased smoothness can be achieved by using more sophisticated higher-order techniques (e.g. cubic splines), however a linear interpolation is enough to link unrolled fringes from the same direction. As a matter of fact, the real difficulty to gain in resolution comes when the target changes its direction.

To keep a good agreement between the reconstructed displacement and calculation resources, we propose to compensate interpolation process in this condition by placing an intermediary coefficient as detailed hereafter.

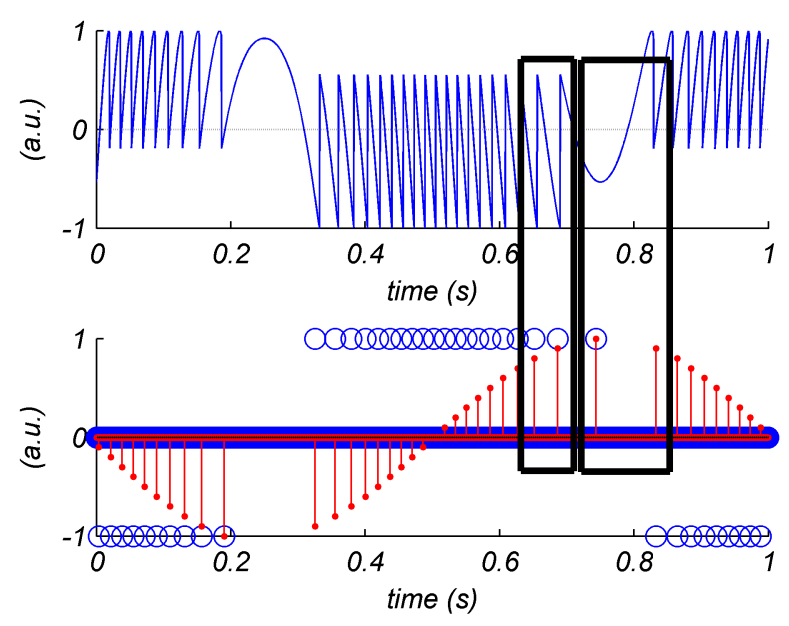

Fig. 7. Principle to obtain sub-wavelength resolution to retrieve target's displacement from irregularly spaced detected fringes. 
Let us now refer to the right block of Fig. 3 to describe the interpolation procedure based on (6). For convenience we have also identified three fundamental calculations for this proposal: 1) transitions pair (TP) exploitation, 2) normal/compensated upsampling, and 3) segmented reconstruction.

1) TP exploitation: Starting from a train of peaks corresponding to temporally detected fringes $(t r)$, TP block parses them and accounts their positive or negative value. It creates a vector with the past $[\operatorname{tr}(N-1)]$ and present $[\operatorname{tr}(N)]$ values where $N=0,1,2, \ldots$, is the number of cumulated fringes. A Counter block keeps track of the number of samples $(T)$ between two detected transitions.

From Fig.7 it can be observed a TP when the target moves in one direction and then when it changes its trajectory. Depending on the sign of TP values, the Zone detector module either selects a Normal upsampling procedure for same-signed transitions, or a Compensated upsampling procedure for unequal signs.

2) Normal/Compensated upsampling: From TP module, if transitions share the same sign (positive or negative), then they are assigned to a Normal zone where simple linear interpolation is carried. The procedure to obtain an upsampled vector is described by:

$$
x(k)= \begin{cases}T P(k / T), & k=0, T \\ 0, & \text { otherwise. }\end{cases}
$$

When TP vector contains a positive value and a negative value or vice-versa, a Compensated zone is assigned requiring a particular interpolation in order to produce a convenient resolution. The procedure to obtain an upsampled vector in this condition is:

$$
x(k)= \begin{cases}T P_{\text {sign }}(k / T) / 2, & k=0, T \\ T P_{\text {sign }}(0)+\varepsilon, & k=T / 2 \\ 0, & \text { otherwise. }\end{cases}
$$

where $\varepsilon$ is a coefficient for resolution adjustment, ideally representing the point where the target changed its direction.

To further define this calculation, we have represented both concepts in Fig.8. The case of a Normal interpolation segment consists in a convolution process between TRI and the upsampled values from TP vector according to (8). Slope segment from the shaded area is thus the interpolation between boundary points 2 and 1 of height. To produce a Compensated interpolation segment respecting the usage of (6) and TRI, the upsampled vector is thus modified according to (9).

Notice that boundary points 0.5 and -0.5 are aimed to cancel themselves through convolution process in the shaded area. This elaboration allows resolution adjustment by adding a coefficient in the range [1-1.5] placed in the middle of the upsampled vector as it pulls up the interpolated slope segment. By using a coefficient equal to 1 , excess fringe coefficient $\varepsilon$ is set to zero leading to a resolution similar to a fringe counting method. A value of 1.25 , provides a behavior equivalent to the proposal in [11] which adds $\pi / 2$ on its elaboration, thus $\varepsilon=\lambda / 8$.

Since this interpolation process happens every two detected fringes, a segment of displacement reconstruction $\mathrm{x}_{\mathrm{FOH}}$ can
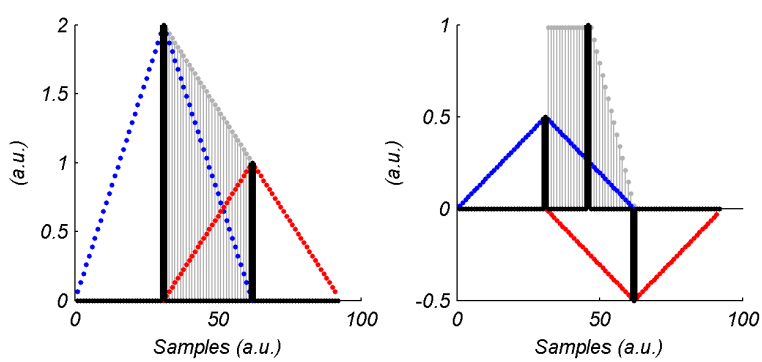

Fig. 8. Interpolation segments (shaded area) obtained by convolution with a triangular impulse response. Normal upsampling results in linear interpolation (left), while Compensated upsampling results in a bumped segment.

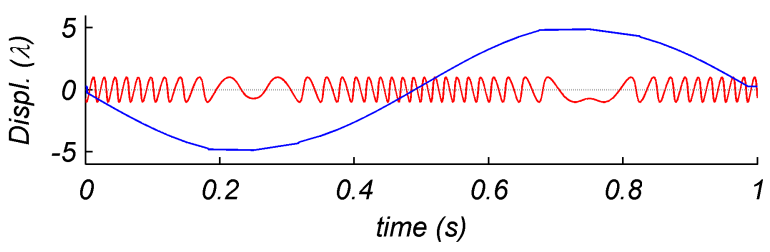

(a) Reconstruction for SM signal with $C=0.5$.

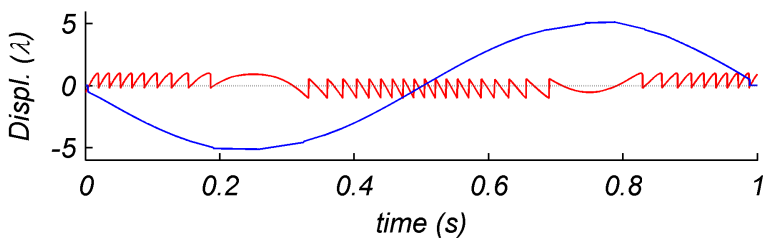

(b) Reconstruction for SM signal with $C=3$.

Fig. 9. Input/output of the proposed real-time versatile algorithm.

be established as a particular case of the ideal interpolation process by:

$$
x_{F O H}(t)=\sum_{k=0}^{T} x(k) h(t-k) .
$$

where $\mathrm{x}(\mathrm{k})$ is an upsampled vector from (8) or (9) and $h(t)$, the generated triangular impulse response.

3) Piece-wise reconstruction: Based on (10) and the relationship between the appearance of a fringe and the amount of displacement, the output of the reconstruction algorithm can be established as:

$$
D(t)=x_{F O H}(t) \frac{\lambda_{0}}{2} .
$$

In Fig.9, this second step has been applied to SM signals of weak and moderate feedback regime to illustrate a complete reconstruction process with $\varepsilon=\lambda / 8$ resolution.

Compared to the referenced work in section II, instead of combining PUM and ATA proposals to obtain a versatile SM displacement measurement sensor, our detectionreconstruction approach does not require iterative (thus expensive) calculations as it does not require to distinguish a particular feedback mode and works in a piece-wise basis. Furthermore, the robust detection step through analytic signals provides an advantage over ATA detection algorithm since it is not affected by speckle phenomenon Following section presents a series of experiments to denote these features. 


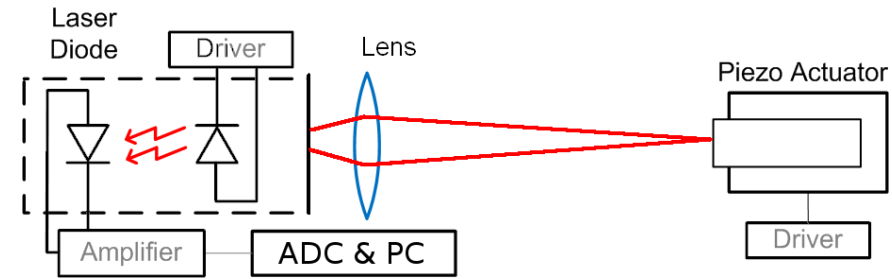

Fig. 10. Experimental setup to acquire SM displacement signals over different surfaces.

\section{EXPERIMENTAL RESULTS}

We performed two kinds of experiments for our algorithm, one to validate its versatility face to optical feedback variations, and the other to validate its feasibility for real-time calculations with different segments of the acquired signal. For the first case we used a piezoelectric transducer (PZT) as vibrating surface providing a counter-measurement of $2 \mathrm{~nm}$ of resolution (Fig.10). Resulting SM signals were recorded with an oscilloscope for post-processing. For the second case we used a mechanical shaker to provide a rough vibration of unknown amplitude. Displacement reconstruction was then performed on each acquired buffer.

A commercial LD (HL7851G) with wavelength $\lambda_{0}=785$ $\mathrm{nm}$ was placed at a distance of about $40 \mathrm{~cm}$ from the target in both cases. To obtain two different feedback levels, we directed the LD over the metallic surface of the PZT and acquired one period of a sinusoidal target movement at $300 \mathrm{~Hz}$ and $\approx 2 \mathrm{Vp}$ p. Then we placed a white paper on its surface and repeated the operation.

\section{A. Moderate feedback}

The obtained OOP variations were observed to fall in moderate regime as attested by the sharpened peaks in one direction of the target and more rounded in the opposite way [Fig.11(a)]. By visual inspection, a total of 20 peaks per period can be accounted for this SM signal, meaning a forwardbackward displacement of $\approx 4 \mu \mathrm{m}$.

In Fig.11(b) the fringe detection step from our algorithm (denoted HT) is compared against the referenced ATA detection proposal. For this case both elaborations provided the same amount of fringes. From them, we have used the same sub-wavelength displacement reconstruction step of our algorithm. As expected, both waveforms are equivalent in amplitude and reproduce the reference displacement signal [Fig.11(c)]. A sample-to-sample error evaluation [Fig.11(d)] allows to account the importance on the position of detected fringes. In the case of HT, a root mean square (RMS) error of $0.249 \mu \mathrm{m}$ has been calculated against $0.453 \mu \mathrm{m}$ of ATA algorithm. Considering only the amount and frequency of reconstructed displacement, it can be stated that both detection algorithms achieved comparable results.

\section{B. Weak feedback}

After covering the PZT with standard printer paper, it was wet with a drop of water to force a dynamic variation on the signal to noise ratio (SNR) within the same experimental

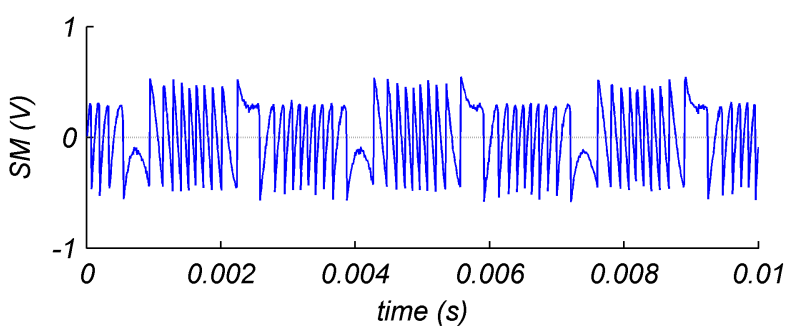

(a) Input SM signal denoting moderate feedback.

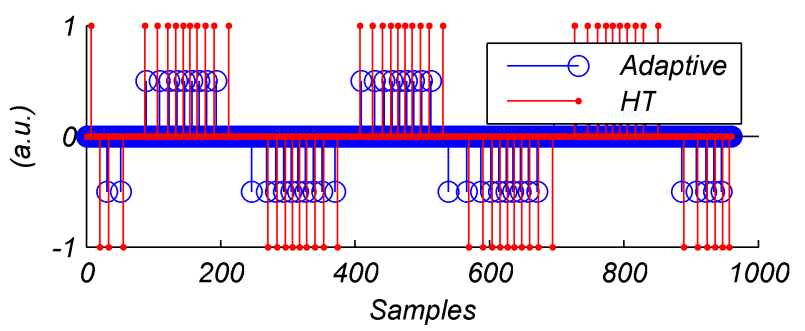

(b) Transitions found by ATA [9] and our proposal (HT).

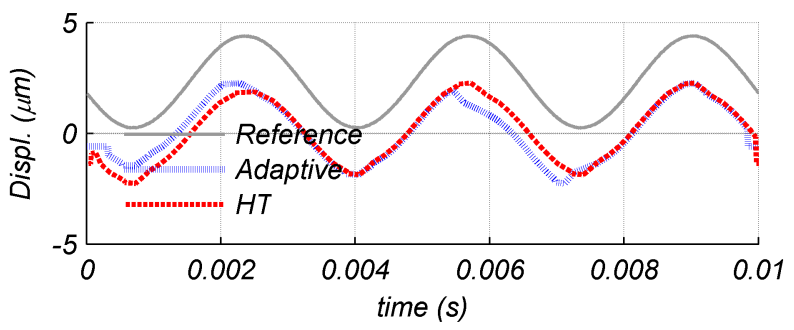

(c) Displacement reconstruction comparison.

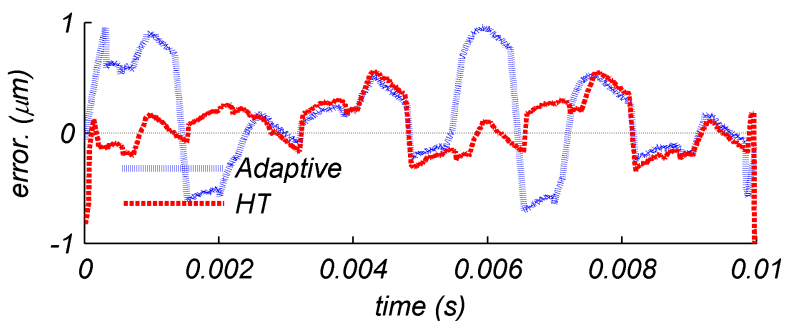

(d) Error between reference and reconstructed displacements.

Fig. 11. Experimental signal obtained from the PZT metallic surface.

conditions. The obtained OOP variations [Fig.12(a)] were observed to fall in weak regime as attested by the rounded peaks and their ambiguity to denote target's direction.

In a first time both algorithms detected all the transitions and assigned them properly in a similar manner as the previous case. However in a second time when SNR decreased, ATA was not able to properly detect the fringes and produced a completely wrong reconstructed segment [Fig.12(b)]. Conversely, our proposal demonstrated a good performance along the time. As shown in the sample-to-sample error comparison [Fig.12(c)], the resolution is kept below the subwavelength requirement with a RMS of error of $0.325 \mu \mathrm{m}$, thus demonstrating that our algorithm provides similar behavior for different feedback conditions.

\section{Real-time implementation}

In order to validate the convenience of implementing our algorithm as a sensor for displacement measurement, we 


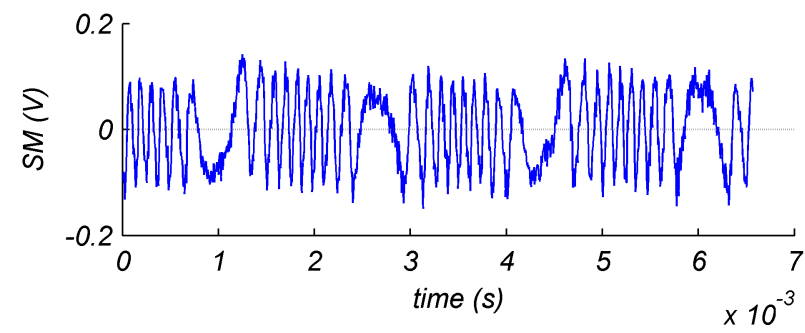

(a) Input SM signal denoting weak feedback.

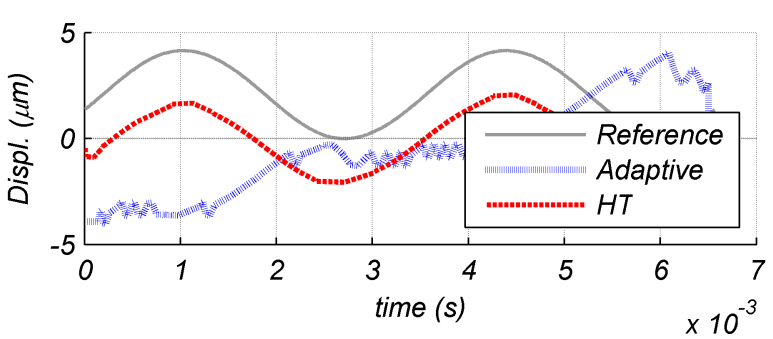

(b) Displacement reconstruction comparison.

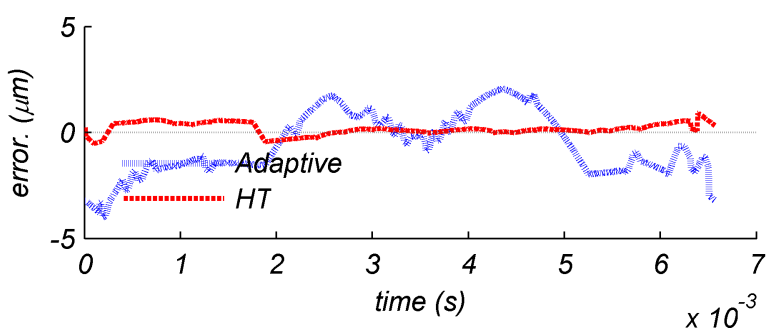

(c) Error between reference and reconstructed displacements.

Fig. 12. Experimental signal obtained from the PZT with a wet white paper on its surface.

implemented our algorithm in Matlab® and added a graphical user interface (GUI). SM signal segments were acquired within a circular buffer via USB by an Analog-to-Digital (A/D) converter card from National Instruments®(USB-6361). Displacement reconstruction was performed in real-time over each acquired signal segment at different boundary times by modifying the sampling rate and buffer size.

Parameters within the GUI are as follows:

a) Sampling rate: Acquisition can be set up to $1 \mathrm{MS} / \mathrm{s}$ in 16 bits resolution.

b) Buffer size: Number of samples to acquire before triggering our algorithm.

c) Denoise input: This is parameter allows to smooth the acquired signal segment. We estimate the number of samples describing a single fringe for a given displacement, this serves to identify the maximum span $(100 \%)$ for an average filter. Depending on the amount of noise observed on the acquired signal, practical span values were changed from 0 to $40 \%$. Notice that this stage should be performed by an electronic filter in a final SM displacement sensor.

d) Pseudo-fringe threshold: This parameter has been introduced to bypass the usage of offset removal in detection step, this is particularly useful in the case of SM signal segments of less than one period of the target's displacement as it will be shown in this section.

e) Direction method: Different fringe direction assignation methods can be evaluated at this level. The method using

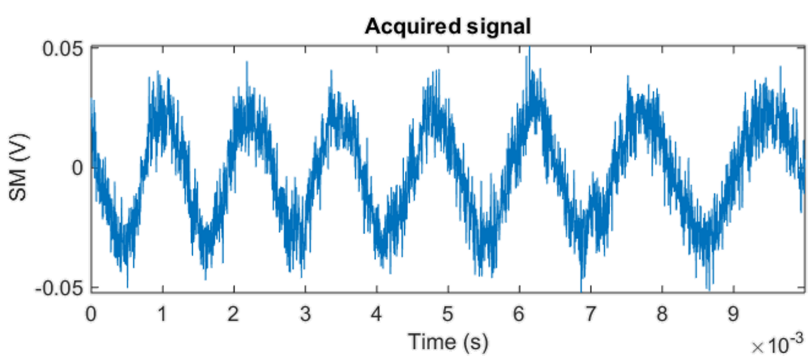

(a) 3000 points of SM signal for a sampling rate of $300 \mathrm{kHz}$.

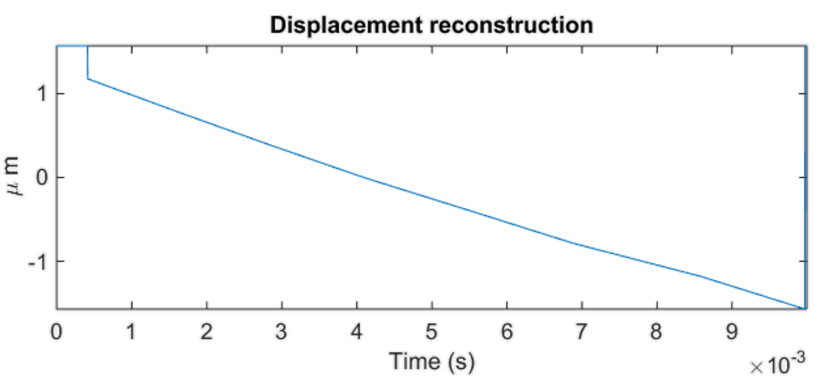

(b) Denoise $=30 \%$, pseudo fringe threshold. $=10 \%$.

Fig. 13. Screenshot of the real-time processing for less than one period of target's displacement.

instantaneous frequency peaks is depicted in Fig.5, however we also implemented the duty cycle calculation from [7].

f) Interpolation bump: This is the coefficient allowing to adjust the resolution as described for equation (9).

The major challenge faced by our algorithm is the identification of the target's direction due to its piece-wise approach. Particularly in the case of non-cooperative targets where the feedback coupling punctually reaches a very weak feedback it is practically impossible to solve the ambiguity just by looking to a single fringe.

First in this experiment we aimed the LD over the shaker driven by a sinusoidal signal at $11 \mathrm{~Hz}$ with $100 \mathrm{mVp}$-p from a function generator. We processed a SM signal segment every $100 \mathrm{~ms}$ and obtained a periodical displacement reconstruction of $\approx 10 \mu \mathrm{m}$ p-p of amplitude similar to previous experiments with the PZT. By setting the sampling rate to $300 \mathrm{kHz}$ with a buffer size of 3000 points less than one period of the target oscillation was acquired. This $10 \mathrm{~ms}$ boundary allowed to establish the minimum real-time constraint in this prototyping platform. Then, we slightly defocused the setup to obtain a noisy SM signal and observe the pertinence of the smooth filter, in this case for $30 \%$ span over a single fringe. As depicted in Fig.13 our algorithm is able to identify the SM fringes in forward direction, meaning that the shaker was moving against the LD in that instant.

Finally we covered the pointed surface of the shaker with a slow-fading phosphor material active in the IR (VRC2 from Thorlabs) to obtain a speckle affected signal. We also changed the target movement to $40 \mathrm{~Hz}$ with $300 \mathrm{mVp}$-p and increased the sampling rate to $500 \mathrm{kHz}$ with a buffer size of 50000 points. This setup required a smooth span of $40 \%$ over a single fringe. As depicted in Fig.14 our algorithm is able to perform displacement reconstruction also in this case. 


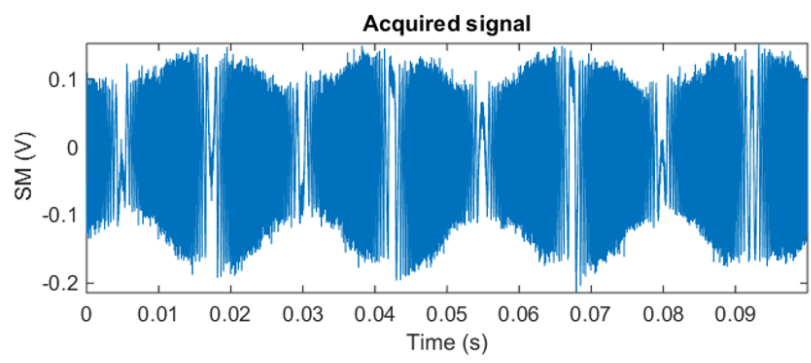

(a) 50000 points of SM signal for a sampling rate of $500 \mathrm{kHz}$.

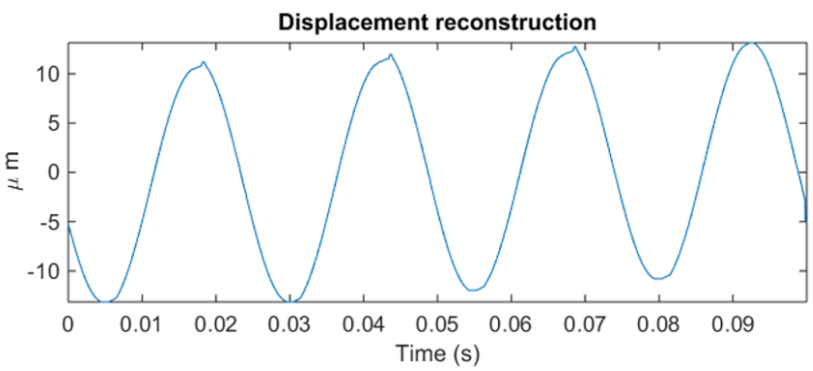

(b) Denoise $=40 \%$ and pseudo fringe threshold $=100 \%$.

Fig. 14. Screenshot of the real-time processing of several periods of target displacement for a speckle affected SM signal.

\section{CONClusion}

We have presented an algorithm to perform displacement reconstruction from SM interferometry signals of different feedback levels. It has been designed to work in a piecewise basis allowing to estimate acquisition boundaries for realtime calculations. The calculation steps rely on basic signal processing functions (FFT, IFFT, Filtering) allowing its implementation over different processing architectures. Compared to another all-purpose algorithm (ATA), our approach can work when less than one period of target's displacement is acquired. We demonstrated improved robustness for a decreased quality of the signal, specially when the amplitude of the fringes changes due to speckle phenomenon. This algorithm performs similarly without any knowledge of the coupling factor, thus resulting convenient for its implementation into an optical sensor suitable to be used in different kinds of conditions and surfaces.

\section{REFERENCES}

[1] S. Donati, "Developing self-mixing interferometry for instrumentation and measurements," Laser Photonics, vol. 6, no. 3, pp. 393 - 417, 2012.

[2] G. Plantier, C. Bes, and T. Bosch, "Behavioral model of a self-mixing laser diode sensor," IEEE J. Quantum Electron., vol. 41, no. 9, pp. $1157-1167$, sep. 2005.

[3] D. Guo, "Quadrature demodulation technique for self-mixing interferometry displacement sensor," Optics Communications, vol. 284, no. 24, pp. 5766-5769, dec. 2011.
[4] G. Giuliani, S. Bozzi-Pietra, and S. Donati, "Self-mixing laser diode vibrometer," Measurement Science and Technology, vol. 14, pp. 24 32, 2003.

[5] O. Bernal, U. Zabit, and T. Bosch, "Robust method of stabilization of optical feedback regime by using adaptive optics for a self-mixing microinterferometer laser displacement sensor," IEEE Journal of Selected Topics in Quantum Electronics, 2014.

[6] S. Merlo and S. Donati, "Reconstruction of displacement waveforms with a single-channel laser-diode feedback interferometer," IEEE $J$. Quantum Electron., vol. 33, pp. 527-531, 1997.

[7] A. Magnani, A. Pesatori, and M. Norgia, "Self-mixing vibrometer with real-time digital signal elaboration," Applied Optics, vol. 51, no. 21, pp. $5318-5325,2012$.

[8] C. Bes, G. Plantier, and T. Bosch, "Displacement measurements using a self-mixing laser diode under moderate feedback," IEEE Transactions on Instrumentation and Measurement, vol. 55, no. 4, pp. 1101-1105, 2006.

[9] U. Zabit, T. Bosch, and F. Bony, "Adaptive transition detection algorithm for a self-mixing displacement sensor," IEEE Sensors Journal, vol. 9, no. 12 , pp. $1879-1886,2009$.

[10] A. Luna Arriaga, F. Bony, and T. Bosch, "Speckle-insensitive fringe detection method based on Hilbert transform for self-mixing interferometry," Applied Optics, vol. 53, no. 30, pp. 6954-6962, 2014.

[11] U. Zabit, T. Bosch, F. Bony, and A. Rakic, "A self-mixing displacement sensor with fringe-loss compensation for harmonic vibrations," IEEE Photon. Technol. Lett., vol. 22, no. 6, pp. 410-412, 2010.

[12] S. L. Marple, "Computing the discrete-time analytic signal via FFT," IEEE Transactions on Signal Processing, vol. 47, pp. 2600-2603, 1999.

[13] J. G. Proakis and D. G. Manolakis, Digital Signal Processing - Principles, Algorithms and Applications. Pearson Prentice Hall, 2007.

Antonio Luna Arriaga received the M.S. degree in instrumentation and systems from the INSA, Lyon, France in 2007. He completed his doctoral studies with the Group of Optoelectronics for Embedded Systems (LAASCNRS), INP of Toulouse, France in 2014. His current post-doctoral research within the same laboratory focuses on signal processing for biomedical sensors based on optical feedback interferometry.

Francis Bony received the $\mathrm{Ph} . \mathrm{D}$ degree in microelectronics, microsystems from the Laboratory for Analysis and Architecture of Systems (LAAS-CNRS), France (2003). In 2005, he became an Assistant Professor in the Electronics Department of ENSEEIHT - INP of Toulouse. He is currently working on digital circuits and electronic system boards for optoelectronic measurement sensors.

Prof. Thierry Bosch got his Ph.D from National Institute of Applied Science of Toulouse (INSAT) in 1992. In 1993, he joined the Department of Automatic Control and Production Systems at the Engineering School of Mines of Nantes, as an Assistant Professor. He is presently professor in the Engineering School ENSEEIHT - INP of Toulouse and at the head of the Research Group on Optoelectronics for Embedded Systems (LAAS-CNRS). His research interests are related to laser industrial instrumentation development including range finding techniques, vibration and velocity measurements. He has cooperated in several programs of R\&D with European companies active in the areas of sensor design, metrology, transportation or avionics. He has co-authored about 50 papers in archival journals and he has been invited to author the chapter dedicated to the optical feedback interferometry published in the Encyclopedia of Sensors edited by American Scientific Publishers in 2006. 